\title{
Internet of Things for Demand Side Management
}

\author{
Giampaolo Fiorentino and Antonello Corsi \\ IoT (Internet of Things) $R \& D$ (Research and Development) Lab, Engineering Ingegneria Informatica S.p.A, Rome 00185, Italy
}

Received: November 24, 2014 / Accepted: January 21, 2015 / Published: May 31, 2015.

\begin{abstract}
The introduction of new kinds of energy mixes to the electricity grid is a challenging environmental task for present and future generations as they fight the pollution and global warming issues associated with urbanization. Individual appliances and whole buildings that continuously incorporate local intelligence which originates from the new technologies of Internet of Things are the new infrastructure that this integration is based on. Smart Electricity Grids are becoming more intensively integrated with tertiary building energy management systems and distributed energy generators such as wind and solar. This new smart network type harnesses the IoT (Internet of Things) principles by generating a new network made of active elements combined with the necessary control and distributed coordination mechanisms. This new self-organized overlay network of connected DER (distributed energy resources) allows for the seamless management and control of the active grid as well as the efficient coordination and exploration of single and aggregated technical prosumer potential (generation and consumption) to participate in energy balancing and other distributed grid related services, applying energy management strategies based on control and predict of the DERs behavior for facing demand side management issues.
\end{abstract}

Key words: Energy, smart grid, aggregator, local hub, demand side management.

\section{Introduction}

Riskless and reliable delivery of energy is essential in our society. Achieving this goal in a world with increasing demand and declining resources is a complicated task. At this purpose, the ongoing restructuring of the old delivery infrastructure need that we move towards increasing the usage of alternative energy resources, which are smaller and decentralized [1].

This conceptual framework leads to a very dynamic future energy network, where electricity will be produced in a distributed way, where customers will be not only consumers but also producers (hence they are called prosumers), and where bidirectional interaction between interested actors will be possible.

The bidirectional exchange of information will put the basis for cooperation among the different entities, and then they ought to be able to access and correlate information that will come from several devices.

The best shape of this new decentralized smart grid

\footnotetext{
Corresponding author: Giampaolo Fiorentino, IoT resercher, research field: energy. E-mail: giampaolo.fiorentino@eng.it.
}

therefore will follow the paradigm of internet of energy [2], where energy flows from suppliers to customers like data packets do in the World Wide Web.

Obviously, these drastic changes on both supply and the demand side: high penetration of both DER (distributed energy resources) and DG (demand generation), will require a sequence of advanced strategies and technologies for maintaining system reliability and flexibility.

\section{Materials and Method}

This new smart grid will be formed by embedded systems, ubiquitous computing and sensor networks.

For this reason, in the context of the EU FP7- ICT (European Commission Seventh Framework Programme-Information and Communication) project INERTIA (integrating active, flexible and responsive tertiary prosumers into a smart distribution grid) [3], a consortium of 10 complementary partners from six different European countries aims at addressing the important challenges that future energy systems will face by building a new modelling 
methodology that integrates physical components and entities of internet of things.

The INERTIA project works on the definition of a new data management infrastructure to allow electricity production and consumption to be measured, reported and controlled, delivering an efficient distribution grid overlay control/management infrastructure (Fig. 1). This new infrastructure will maximize the response capacity of the vast, small-commercial prosumer base (e.g., tertiary buildings, offices, etc.) to energy needs presenting incentives and delivering benefits through their automated active participation in the energy market.

\subsection{Inertia Framework}

Within INERTIA, distributed energy resources will form self-organised networks of intelligent active nodes that efficiently distribute and balance global and local energy. This self-organized overlay network allows for the seamless management and control of the active grid as well as efficient coordination between suppliers (traditional RES (renewable energy sources)) and prosumers (local generation and consumption). To this end, the overall control network consist of two types of peers /hubs, while, agent subcomponents run on both hubs, underlying the self-organised distributed grid and load control operations.

The first type of hub is the aggregator control hub that will manage the prosumer portfolio, treading with market stakeholders on behalf of small customers. Aggregators moreover gather, analyse and efficiently organize their customer load portfolio's and define specific AD (active demand) strategies and services based on market needs.

Summarizing they act as an intermediary between suppliers and network operators and the different commercial and industrial prosumers belonging to their portfolio.

The second level is constituted from the local control and automation hub that corresponds to the level of building areas. Local control actions and information at this purpose are automatically managed in real-time by

Internet of Things + Smart Grid = Internet of Energy

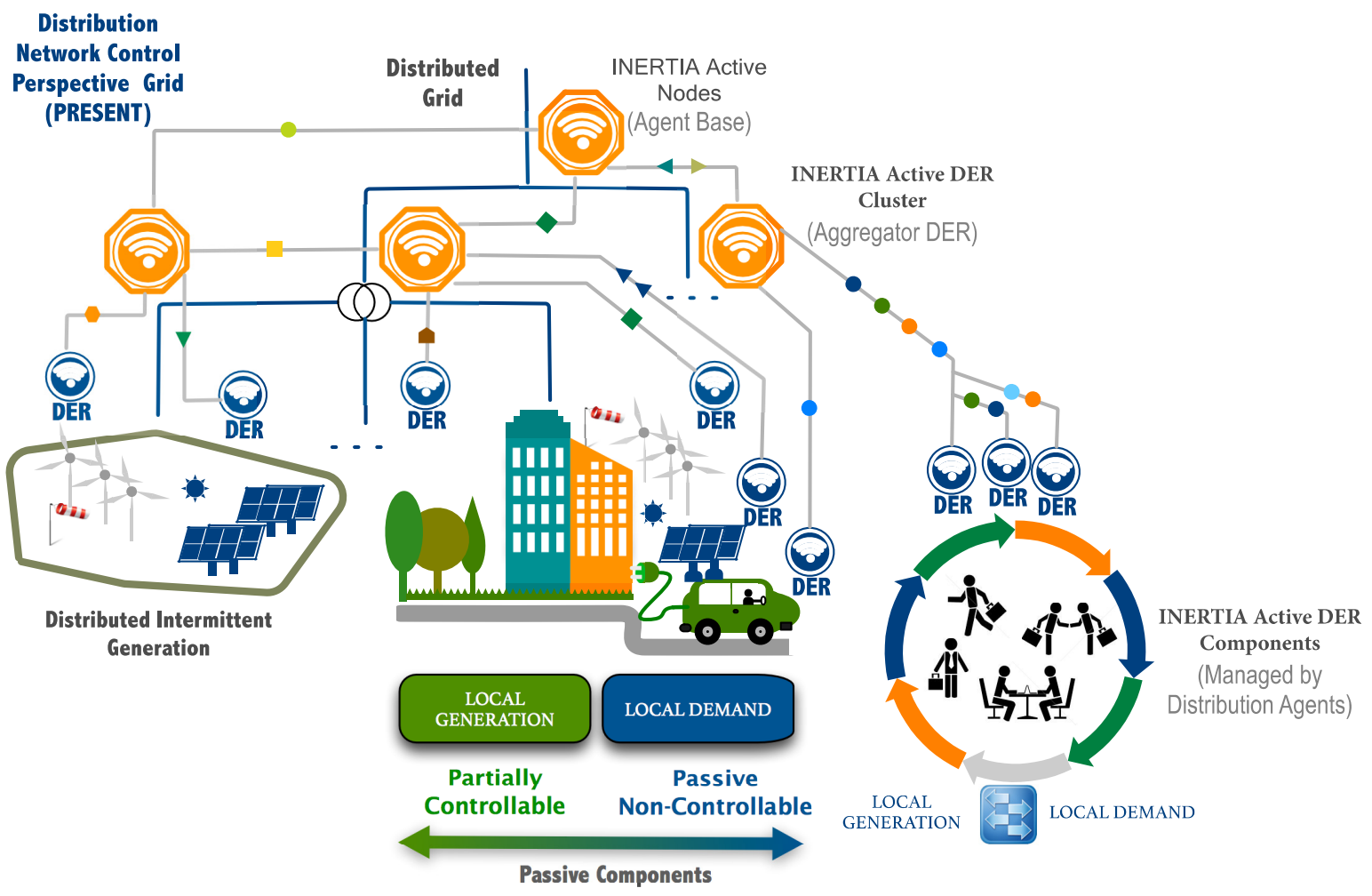

Fig. 1 INERTIA concept. 
the local control hub. This minimize the required interaction by the prosumers and ensure self-privacy and autonomy of tertiary buildings by delivering a self-learning building hub that models the building and occupancy operations as an active aspect of the building.

This architecture can allow exploit load flexibility offered by distributed and autonomous intelligent commercial (tertiary buildings) prosumer hubs and react to specific grid events or market opportunities through the implementation of advanced DR (demand response) strategies. Thus, INERTIA should examine both local (i.e., tertiary buildings) and global (i.e., aggregator, system operators) needs in order to provide a holistic coordination environment where both local hub and aggregator hub coexist under either competitive or cooperative behaviors.

\subsection{Inertia Operation}

The wide deployment of renewable DER technologies will significantly modify the demand profile of power systems and will affect the way distribution grids are managed and operated [4]. A high penetration of DER however, can significantly affect the network operation in a number of ways. The main operational issues are: violation of thermal limits; overvoltage issues; increased fault currents exceeding the short circuit capacity of the network; increased harmonic emissions and reverse power flow causing negative effects on certain types of tap changers and on the operation of voltage control and protection schemes.

Due to these adverse effects on distribution network operation, DSOs (distribution system operators) many times adopt conservative measures for RES deployment and are reluctant to allow a high penetration of DER [5].

INERTIA can fix all of this takes into account how demand response can change the end user comfort.

Using a Bayesian formalism that is applied to infer the probability that any environmental situation should be considered by the user as environmentally uncomfortable, and defining the upper and lower limits of the energy flexibility that can be offered to the whole energy system, INERTIA will be able to safeguard the electricity grid, facing a trade-off between energy provided by the local generation facility and consumers' consumption. In this respect, the holistic flexibility model generates the suitable energy demand strategy deploying DSM (demand side management) operations, which takes into account the final users comfort. The comfort is evaluated also by the facility manager selected to enroll a specific DR program.

\section{Results}

Let to apply INERTIA framework to the following use case: use of flexibility to avoid voltage limit violations in a network with high penetration of distributed generation.

Following a prediction for sunny and windy weather, the framework can infer that renewable energy production can be more than energy consumption. This info can flow into energy production plans of aggregator that can select the best local hub portfolio able to schedule energy hungry tasks during that time: It can decide to fully charge electric cars, postpone dishwasher, dimming light and turn off HVAC (heating, ventilating and air conditioning).

Due to the dynamic distributed nature of smart grid, as well as its large scale, optimizations will result at local level, and negotiations and cooperation among all entities will eventually lead to energy efficiency with estimated result in a general case of reducing the cost per consumer from $€ 5.8 /$ year to $€ 32.3 /$ year.

Reducing balancing costs, price volatility and higher use of renewable generator leads to reduce the cost per consumer from $€ 0.7 /$ year to $€ 3.8 /$ year.

Reducing network losses by reducing congestions and adapting better the network capacity reducing the 
cost per consumer by $€ 0.5 /$ year to $€ 3.3 /$ year.

Reducing network investments reducing peak load and therefore network reinforcements reducing the cost per consumer by $€ 0.15 /$ year to $€ 9.4 /$ year.

\section{Conclusions}

New IoT (Internet of Things) technologies are essential for implementing electrical power systems in smart cities and reducing environmental impacts and social costs. At the moment, appliances can self-adjust to consume less power or even turn themselves off. Using the data collected from all the devices, algorithms can calculate the exact energy demand so to reduce the need for standby generation. Over time, generation can be automatically shifted according to predicted increases and decreases in demand. Moreover, a framework that receives input from relevant sources such as weather information, heating, cooling, lighting equipment and usage scenarios (occupancy rates) can be implemented to compute energy consumption and identify available flexibilities from DER. This could be conducted in a non-invasive way that would respect the comfort of the end user.

\section{References}

[1] Buccella, C., Cecati, C., and Abu-Rub, H. 2014. "An Overview on Distributed Generation and Smart Grid Concepts and Technologies." In Power Electronics for Renewable Energy Systems, Transportation and Industrial Applications, edited by Abu-Rub, H., Malinowski, M., and Al-Haddad, K. Chichester: John Wiley \& Sons, Ltd.

[2] Hua, L., Junguo, Z., and Fantao, L. 2014. "Internet of Things Technology and Its Applications in Smart Grid." TELKOMNIKA Indonesian Journal of Electrical Engineering 12 (2): 940-6.

[3] EU (European Union). 2015. "INERTIA: Integrating Active, Flexible and Responsive Tertiary Prosumers into a Smart Distribution Grid." EU.

[4] Palensky, P., and Dietrich, D. 2011. "Demand Side Management: Demand Response, Intelligent Energy Systems, and Smart Loads." IEEE Transactions on Industrial Informatics 7 (3): 381-8.

[5] De Joode, J., Jansen, J. C., Van der Welle, A. J., and Scheepers, M. J. J. 2009. "Increasing Penetration of Renewable and Distributed Electricity Generation and the Need for Different Network Regulation." Energy Policy 37 (8): 2907-15. 